\section{Acute obstructive cholangitis caused by barium refluxed into the common bile duct through endo- prosthesis}

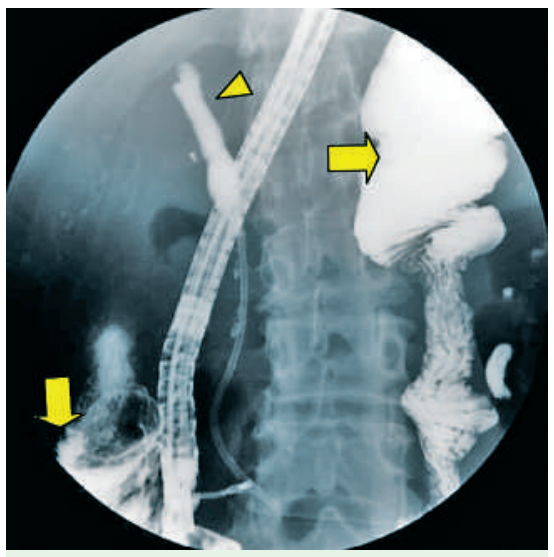

Fig. 1 Radiograph image taken at retrieval of endoprosthesis before cholangiogram. An arrowhead indicates barium of the common bile duct. Arrows indicate remained barium of transverse colon 6 days after an upper gastrointestinal series.

A 65-year-old man was hospitalized because of early gastric cancer (UICC, stage II) and pancreatic cancer (UICC, Stage III). An endoprosthesis, $7 \mathrm{Fr}$ in size, had been placed for obstructive jaundice due to pancreatic cancer in another hospital. Laboratory data on admission showed slight elevation of total bilirubin $(1.7 \mathrm{mg} /$
dL). High-grade fever and remarkable jaundice developed (total bilirubin, $6.1 \mathrm{mg} / \mathrm{dL}$ ) 6 days after an upper gastrointestinal series for evaluation of gastric cancer. We considered acute obstructive cholangitis due to occlusion of the endoprosthesis, and performed endoscopic retrograde cholangiopancreatography. A radiograph taken while retrieving the endoprosthesis prior to a cholangiogram showed barium at the common bile duct as well as transverse colon ( $\bullet$ Fig. 1). Although we tried to remove the barium by basket catheter after retrieving the endoprosthesis, the basket catheter could not grasp enough of the barium ( $\bullet$ Fig. 2 a). In spite of the presence of a biliary stricture due to pancreatic carcinoma, the balloon catheter was able to remove the barium to the distal side of the stricture little by little. Surprisingly, however, inflation maneuver of the balloon catheter resulted in migration of the proximal barium into the biliary trees ( $\nabla$ Fig. $\mathbf{2 b}$ ). Finally, a 7 Fr nasobiliary drainage catheter was placed ( $\bullet$ Fig. 2c). It took 4 days for the nasobiliary drainage catheter to completely clean the barium from the biliary trees ( $\bullet$ Fig. 2d).
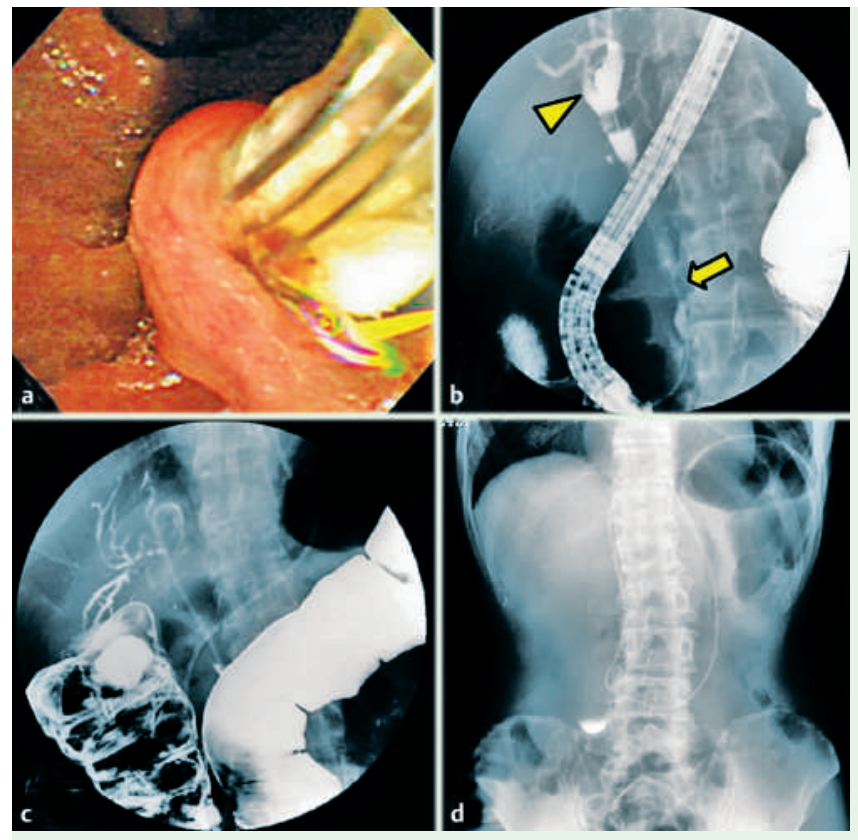

Fig. 2 An endoscopic view and radiographs. a Basket catheter can grasp a little amount of barium. b Balloon catheter can retrieve the barium in spite of the biliary stricture (arrow). Simultaneously, inflation of balloon catheter leads to migration of barium into proximal biliary trees (arrowhead). c Radiograph immediately after placement of nasobiliary catheter to drain the migrated barium. $\mathbf{d}$ An abdominal radiograph 4 days after placing the nasobiliary drainage catheter. The migrated barium has disappeared completely.
The reason why barium refluxed into the common bile duct through endoprosthesis remains unknown. This complication is extremely rare and has not been reported before. In the patients who have a resectable tumor, placement of a $7 \mathrm{Fr}$ nasobiliary drainage catheter might be the first choice for obstructive jaundice by barium through endoprosthesis, because the basket catheter is not effective and the balloon catheter leads to migration of barium into proximal biliary trees. On the contrary, in patients who have an unresectable tumor, placement of a selfexpandable metallic stent would be a better choice.

To avoid mistreatment, endoscopists should be aware of this extremely rare barium-related complication.

\section{Endoscopy_UCTN_Code_CPL_1AK_2AD}

\section{T. Yoshino, S. Yazumi, T. Chiba}

Department of Gastroenterology \&

Hepatology, Graduate School of Medicine, Kyoto University, Kyoto, Japan

\section{Bibliography}

DOI $10.1055 / \mathrm{s}-2007-966795$

Endoscopy 2007; 39: E315

(c) Georg Thieme Verlag KG Stuttgart · New York . ISSN 0013-726X

\section{Corresponding author}

\section{S. Yazumi, MD}

Department of Gastroenterology \& Hepatology

Graduate School of Medicine

Kyoto University

54 Kawahara-cho

Shogoin

Sakyo-ku

Kyoto, 606-8507

Japan

Fax: +81-75-751-4303

yazoo@kuhp.kyoto-u.ac.jp 